\title{
Effectiveness of Inspiratory Muscle Training on Respiratory Muscle Strength in Patients Undergoing Cardiac Surgeries: A Systematic Review With Meta-Analysis
}

\author{
Fiona Verdine Dsouza, MPT ${ }^{1}$, Sampath Kumar Amaravadi, $\mathrm{PhD}^{1,2}$, \\ Stephen Rajan Samuel, $\mathrm{PhD}^{1}$, Harish Raghavan, $\mathrm{MCh}^{3}$, Nagaraja Ravishankar, $\mathrm{PhD}^{4}$
}

\footnotetext{
${ }^{1}$ Department of Physiotherapy, Kasturba Medical College, Mangalore, Manipal Academy of Higher Education, Manipal, Karnataka, India;

${ }^{2}$ Department of Physiotherapy, College of Health Sciences, Gulf Medical University, Ajman, United Arab Emirates;

${ }^{3}$ Department of Cardiothoracic Surgery, Kasturba Medical Hospital, Mangalore, Karnataka, India;

${ }^{4}$ Department of Biostatistics, Vallabhbhai Patel Chest Institute, University of Delhi, New Delhi, India
}

To determine the effect of inspiratory muscle training (IMT) on pulmonary function, respiratory muscle strength (RMS), and functional capacity in patients undergoing cardiac surgery. The PubMed, PEDro, CINAHL, Web of Science, CENTRAL, and EMBASE databases were searched from inception to June 2020. Randomized controlled trials (RCTs) that evaluated patients who underwent cardiac surgery were included in this review. Meta-analysis performed using a random-effects model showed that the mean difference in forced vital capacity, forced expiratory volume in 1 second, 6-minute walk distance, and RMS was 3.47\% (95\% confidence interval [CI], 0.57 to 6.36 ), $5.80 \%$ (95\% CI, 2.03 to 9.56), $78.05 \mathrm{~m}$ (95\% CI, 60.92 to 95.18), and $4.8 \mathrm{cmH}_{2} \mathrm{O}$ (95\% CI, -4.00 to 13.4 ), respectively. There is strong evidence that IMT improves inspiratory muscle strength, pulmonary function, and functional capacity, and reduces the length of hospital stay in patients undergoing cardiac surgery.

Keywords Cardiac rehabilitation, Respiratory muscle training, Cardiac surgical procedures, Exercise tolerance

\section{INTRODUCTION}

Cardiovascular diseases, especially coronary heart disease, have become the main cause of morbidity and mortality in India [1]. In 2017, 17.8 million deaths due to cardiovascular diseases were reported globally [2].

Received February 3, 2021; Revised April 6, 2021; Accepted May 17, 2021; Published online August 30, 2021

Corresponding author: Sampath Kumar Amaravadi

Department of Physiotherapy, College of Health Sciences, Gulf Medical University, Ajman 4184, United Arab Emirates. Tel: +971-543204752, Fax: +971-67431222, E-mail: sampathkpt@gmail.com

ORCID: Fiona Verdine Dsouza (https://orcid.org/0000-0003-3921-6805); Sampath Kumar Amaravadi (https://orcid.org/0000-0002-4744-0180); Stephen Rajan Samuel (https://orcid.org/0000-0002-5636-2620); Harish Raghavan (https://orcid.org/0000-0002-2926-2181); Nagaraja Ravishankar (https://orcid.org/0000-0002-9533-039X).

(c) This is an open-access article distributed under the terms of the Creative Commons Attribution Non-Commercial License (http://creativecommons.org/ licenses/by-nc/4.0) which permits unrestricted noncommercial use, distribution, and reproduction in any medium, provided the original work is properly cited. Copyright ( 2021 by Korean Academy of Rehabilitation Medicine 
Worldwide, coronary artery bypass graft (CABG) surgery is performed daily in patients with coronary artery disease [3]. Valve repair or replacement is the treatment of choice for patients with valve diseases [4].

Despite advances in surgical procedures, the risk of postoperative complications still exists, as surgeries are now being performed on high-risk patients [5]. It is well known that cardiac surgery causes postoperative pulmonary dysfunction [6], which results in increased effort for breathing, hypoventilation, ineffective cough, and hypoxemia, which leads to postoperative pulmonary complications (PPCs), such as atelectasis, bronchospasm, pneumonia, respiratory failure, acute respiratory distress syndrome, prolonged mechanical ventilation, diaphragmatic dysfunction, and pleural disorders $[7,8]$.

Respiratory muscle dysfunction is a consequence of numerous factors. The grafts harvested for CABG, specifically the internal mammary artery graft, reduces blood supply to the intercostal muscles, which leads to respiratory muscle weakness $[9,10]$. The median sternotomy incision results in pain and altered chest wall mechanics. Sedation and pain together can result in shallow breathing and hypoventilation. All these reduce pulmonary function $[9,11]$.

In addition, the use of cardiopulmonary bypass, topical cooling for myocardial protection (phrenic nerve injury) [6], use of chest drains, especially intercostal drains, further reduces pulmonary function [9]. Even after 1 year, the pulmonary function remains reduced and is not restored to preoperative levels, although the reduction is lesser than that after 4-5 months; compared with the $6 \%-13 \%$ reduction in pulmonary function at 4-5 months, there is a reduction of $4 \%-5 \%$ at 1 year [12]. Pulmonary dysfunction is significant in the early postoperative period, and is the primary cause of PPCs, morbidity, and mortality. However, the clinical significance of decreased pulmonary function at 1 year postoperatively is not known [12,13].

Inspiratory muscle training (IMT) targets the respiratory muscles by increasing the load to inspiration, thereby increasing strength and endurance. Inspiratory muscles respond in a manner similar to that of the other skeletal muscles. IMT has been found to increase strength in healthy individuals, mechanically ventilated patients, and patients with chronic heart failure [14]. Many interventions, such as early mobilization, diaphragmatic breathing exercises, and the active cycle of breathing technique, are traditionally practiced postoperatively in the intensive care unit to prevent the risk of postsurgical pulmonary complications. In addition, devices such as positive expiratory pressure devices, IMT devices, and incentive spirometers are used to reduce the incidence of PPCs after cardiac surgeries $[7,15]$. Prerehabilitation also plays a vital role as it assists the individual to withstand predicted cardiorespiratory and musculoskeletal challenges postcardiac surgery [14]. Mobilization of the extremities; IMT; and IMT using a device that includes isocapnic/normocapnic hyperpnea training, inspiratory resistive flow training, inspiratory threshold pressure training, and incentive spirometry is part of prehabilitation [14]. IMT can also be used in phase II of cardiac rehabilitation along with aerobic training or resistance exercises in patients with chronic heart failure and chronic obstructive pulmonary disease, as evidence has shown improvements in respiratory muscle strength (RMS) in this population [3].

The purpose of this systematic review was to determine the effects of IMT on RMS, pulmonary function, and functional capacity in individuals undergoing cardiac surgery.

\section{MATERIALS AND METHODS}

The present systematic review and meta-analysis was conducted following the Preferred Reporting Items for Systematic Review and Meta-analysis (PRISMA) guidelines. This systematic review protocol was registered in PROSPERO on July 7, 2020 (Registration No. CRD42020179130) and the Center for Open Science (OSF) registry (https://doi.org/10.17605/OSF.IO/U6VNM).

\section{Search strategy}

A comprehensive literature search was conducted in the PubMed, PEDro, CINAHL, Web of Science, CENTRAL, and EMBASE databases from inception to June 2020. The following keywords were used for the search: "cardiac surgeries," "cardiac surgical procedures," "inspiratory muscle training," "respiratory muscle training," "preoperative physiotherapy," "preoperative care," "respiratory muscle strength," "CABG," "coronary artery bypass grafting," "valve replacement," "heart valve replacement surgery," "congenital disability," "congenital disability 
correction," "functional capacity," and "cardiac rehabilitation." The Boolean operators used were "AND," "OR," and "NOT." The search was limited to articles published in English.

\section{Eligibility criteria and study selection}

All randomized controlled trials that evaluated patients who underwent cardiac surgeries and were given IMT, either preoperatively or postoperatively, were included in this review.

The outcomes that had to be assessed were forced expiratory volume in 1 second $\left(\mathrm{FEV}_{1}\right)$, forced vital capacity (FVC), maximal inspiratory pressure (MIP), maximal expiratory pressure, and 6-minute walk test. The secondary outcomes to be investigated were the length of hospital stay and the incidence of PPCs.

For the study to be eligible, (1) it should have enrolled adults above 18 years of age, regardless of their gender and (2) it should have at least one outcome measure considered for this systematic review.

The studies were excluded for the following reasons: (1) studies with incomplete data (data in the form of graphs), (2) studies published in languages other than English, (3) studies that did not evaluate any of the outcomes consid- ered for our systematic review, and (4) studies on neonates and children

The selected studies were downloaded from Mendeley, and retrieved articles were screened for duplicates by two authors (ASK and FVD). For articles that were eligible based on their titles and abstracts, the full text was obtained and re-evaluated to verify whether they met the inclusion criteria. The process of screening and assessment of retrieved studies for inclusion in the systematic review and meta-analysis is presented in Fig. 1.

\section{Data extraction}

A data extraction template was developed, pilot tested, and modified. Data extracted included author names, study title, objective, design, participant characteristics, and intervention details. The authors were contacted if the outcome data were unclear or not reported. Data were extracted independently by one researcher, while another researcher cross-checked the same, and the following information was retrieved: the age of the participants included in the study, the type of IMT given, the number of days of preoperative or postoperative intervention, the intensity and time duration per session, and the outcome measures assessed in each study (both

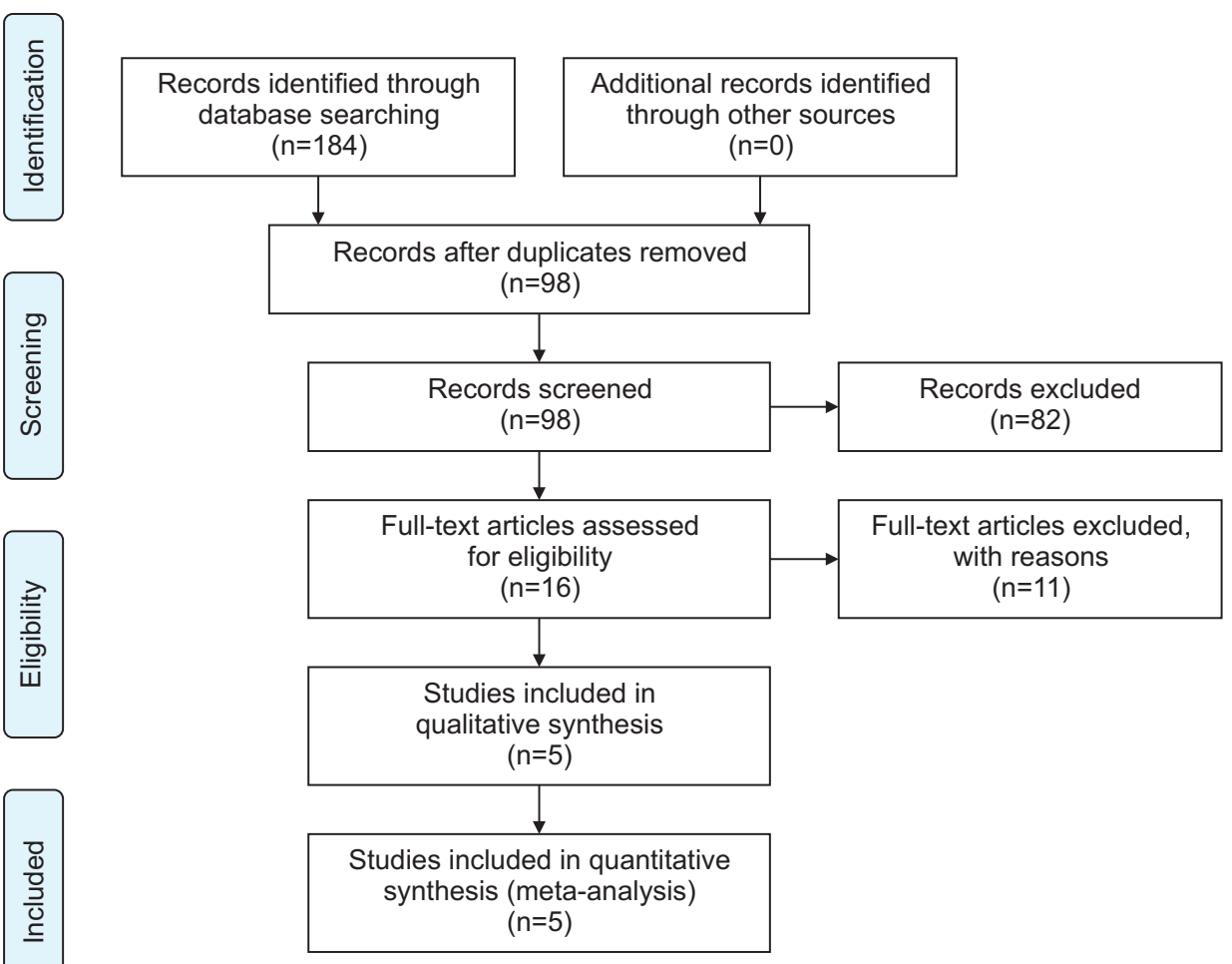

Fig. 1. The Preferred Reporting Items for Systematic Review and Meta-analysis (PRISMA) flow chart. 


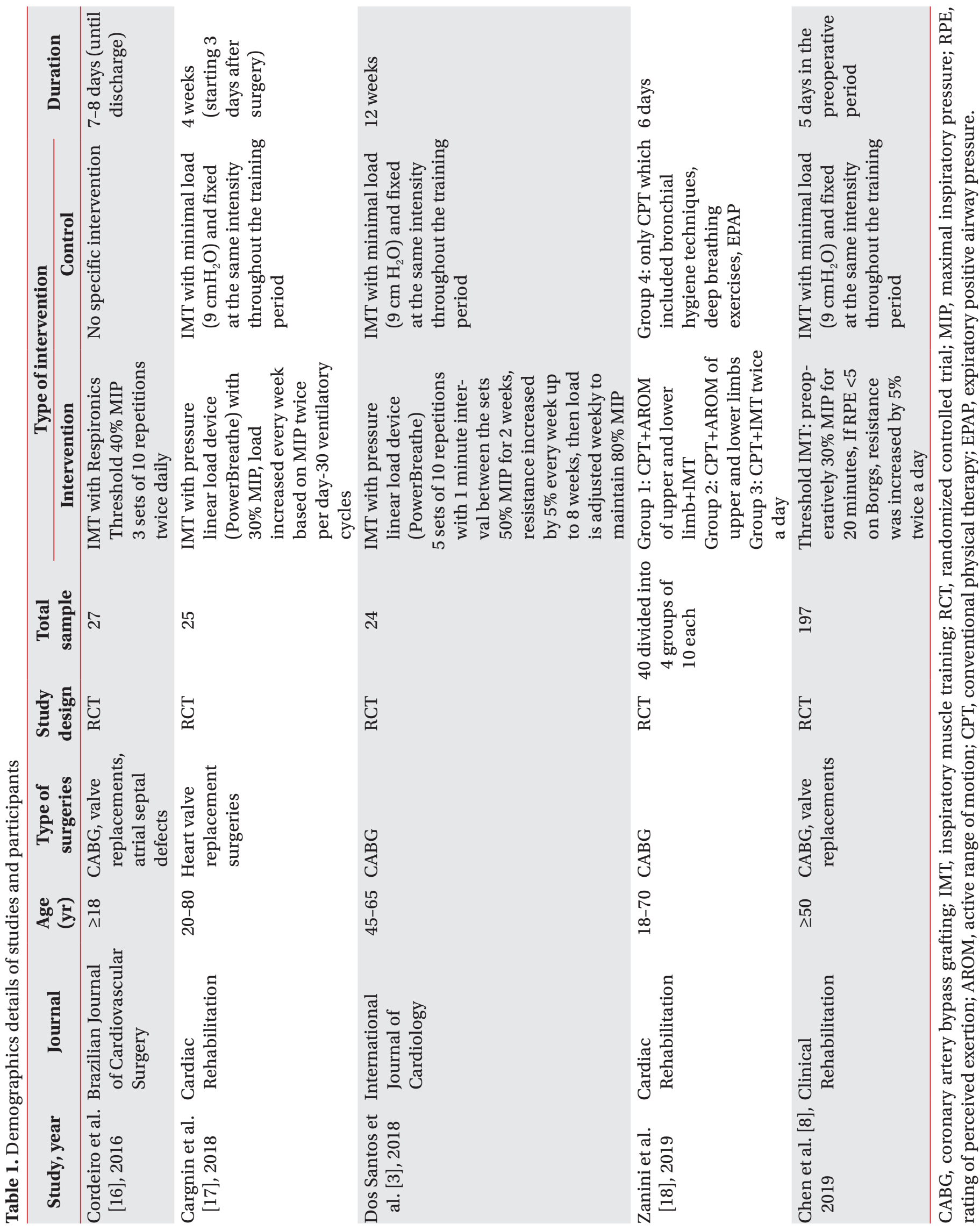


primary and secondary). The details of each study are summarized in Table 1.

\section{Assessment of risk of bias}

The risk of bias for studies included in this systematic review was assessed using the modified Downs and Black checklist, as shown in Table 2 . This checklist provides an overall score of 27 , representing reporting quality, internal validity (confounding bias), power, and external validity. The items are rated as 1 for "Yes" and 0 for "No" or "unable to determine."

\section{Data synthesis}

Meta-analysis was performed using random effects model when at least two studies were similar in terms of the population, intervention, comparison, and outcomes process and study design. As all the outcomes were continuous, mean difference was computed as the effect measure. The chi-square statistic was used $(\mathrm{p}<0.1$ was considered statistically significant) to assess heterogeneity and the $\mathrm{I}^{2}$ statistic (value $>60 \%$ was considered substantial heterogeneity) was used to quantify heterogeneity as percentage. RevMan 5.2 software was used for meta-analysis. We present forest plots for all meta-analy-

Table 2. Downs and black checklist

\begin{tabular}{|c|c|c|c|c|c|}
\hline $\begin{array}{c}\text { Downs and black } \\
\text { checklist }\end{array}$ & Cordeiro et al. [16] & Cargnin et al. [17] & Dos Santos et al. [3] & Zanini et al. [18] & Chen et al. [8] \\
\hline 1 & Yes & Yes & Yes & Yes & Yes \\
\hline 2 & Yes & Yes & Yes & Yes & Yes \\
\hline 3 & Yes & Yes & Yes & Yes & Yes \\
\hline 4 & Yes & Yes & Yes & Yes & Yes \\
\hline 5 & Yes & Yes & Yes & Yes & Yes \\
\hline 6 & Yes & Yes & Yes & Yes & Yes \\
\hline 7 & Yes & Yes & Yes & Yes & Yes \\
\hline 8 & No & Yes & Yes & No & Yes \\
\hline 9 & Yes & Yes & Yes & Yes & Yes \\
\hline 10 & Yes & Yes & Yes & Yes & Yes \\
\hline 11 & Yes & Yes & Yes & Yes & Yes \\
\hline 12 & Yes & Yes & Yes & Yes & Yes \\
\hline 13 & UTD & UTD & No & UTD & UTD \\
\hline 14 & No & Yes & Yes & No & Yes \\
\hline 15 & No & Yes & Yes & Yes & Yes \\
\hline 16 & Yes & Yes & Yes & Yes & Yes \\
\hline 17 & Yes & Yes & Yes & Yes & Yes \\
\hline 18 & Yes & Yes & Yes & Yes & Yes \\
\hline 19 & UTD & Yes & Yes & Yes & Yes \\
\hline 20 & Yes & Yes & Yes & Yes & Yes \\
\hline 21 & Yes & Yes & Yes & Yes & Yes \\
\hline 22 & Yes & Yes & Yes & Yes & Yes \\
\hline 23 & Yes & Yes & Yes & Yes & Yes \\
\hline 24 & No & Yes & No & UTD & Yes \\
\hline 25 & No & UTD & Yes & Yes & Yes \\
\hline 26 & No & Yes & Yes & Yes & Yes \\
\hline 27 & No & Yes & Yes & Yes & Yes \\
\hline Total score & 19 & 25 & 25 & 23 & 26 \\
\hline
\end{tabular}

UTD, unable to determine. 
ses. When the meta-analysis was not possible, the mean difference with $95 \%$ confidence interval (CI) is presented.

\section{RESULTS}

The computerized database search retrieved 184 articles, of which 16 were selected for full-text review, and five were included in the final review.

Articles were excluded based on study methodology, languages other than English, outcome measures other than those to be analyzed in the review, and articles with inadequate data on outcome measures. Data from 293 participants were analyzed. The intervention and control groups included 144 and 149 patients admitted for cardiac surgery, respectively. All participants were older than 18 years. The characteristics of the participants are presented in Table 1. Participants were recruited from hospitals or outpatient clinics.

\section{Functional capacity}

Four studies assessed functional capacity as measured by the 6 -minute walk test as an outcome $[3,16-18]$. There were 46 participants in the intervention group and 150 in the control group. The heterogeneity $\left(\mathrm{I}^{2}\right)$ was $0 \%\left(\mathrm{P}_{\text {Het- }}\right.$ erogeneity $=0.53$ ). Compared to the control group, the intervention group showed significant improvement in the 6-minute walk distance with a mean difference of 78.05 m (95\% CI, 60.92 to 95.18) (Fig. 2).

\section{Pulmonary function}

FVC

Two studies analyzed FVC $[8,18]$. A total of 108 and 109 participants were included in the intervention and control groups, respectively. The heterogeneity $\left(\mathrm{I}^{2}\right)$ was $0 \%$ $\left(\mathrm{P}_{\text {Heterogeneity }}=0.72\right)$. These studies reported significant improvement in FVC in the intervention group compared to that in the control group, with a mean difference of $3.47 \%$ (95\% CI, 0.57 to 6.36) (Fig. 3).

\section{FEV $V_{1}$}

Two studies analyzed $\mathrm{FEV}_{1}[8,18]$. The intervention and control groups comprised of 108 and 109 participants, respectively. The heterogeneity $\left(\mathrm{I}^{2}\right)$ was $0 \%\left(\mathrm{P}_{\text {Heterogene- }}\right.$ ity $=0.20$ ). These studies reported remarkable improvement in $\mathrm{FEV}_{1}$ with a mean difference of $5.80 \%$ (95\% CI, 2.03 to 9.56 ) (Fig. 4).

\section{FEV $V_{1} /$ FVC ratio}

One study reported the effect of IMT on the $\mathrm{FEV}_{1} / \mathrm{FVC}$ ratio [8]. There were 98 and 99 participants in the intervention and control groups, respectively. There was a mean difference of 0.47 (95\% CI, 2.37 to 3.31$)$.

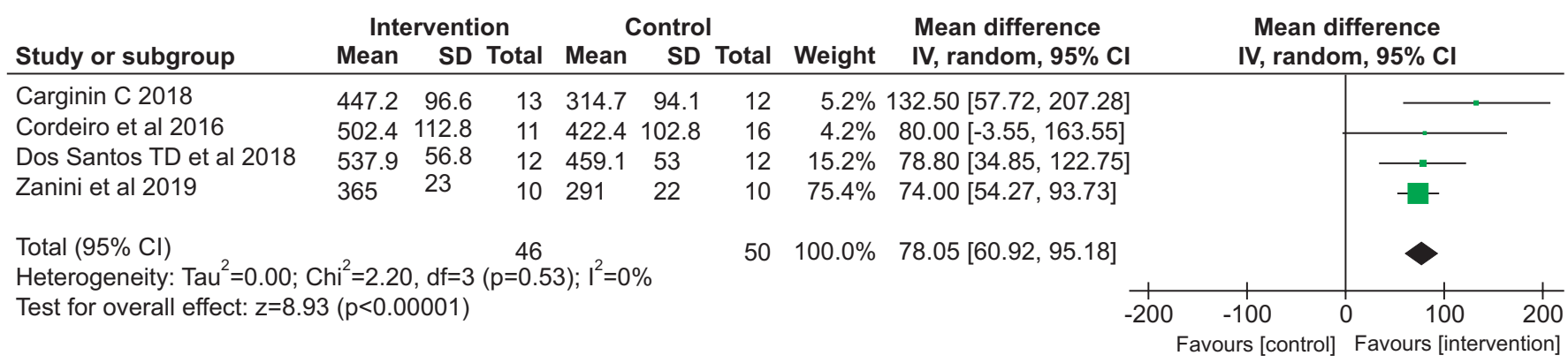

Fig. 2. Forest plot comparing the intervention group versus control group in the 6-minute walk distance.

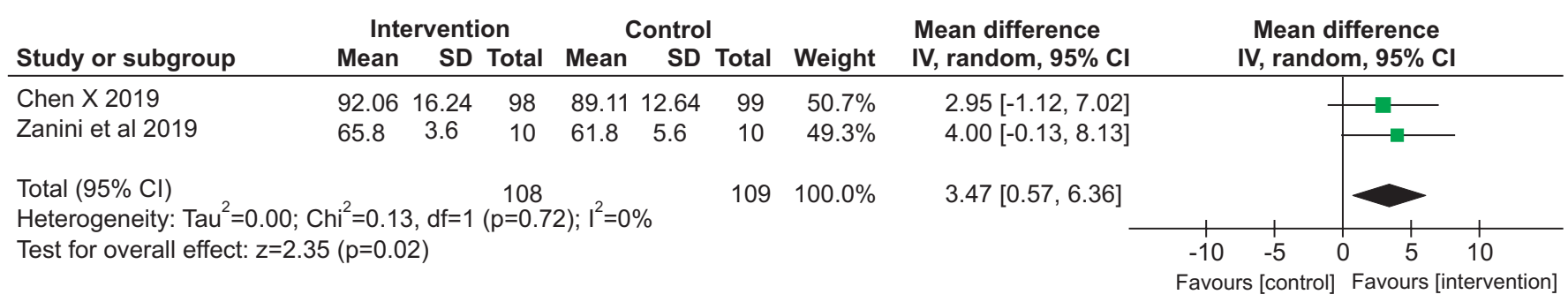

Fig. 3. Forest plot comparing the intervention group versus control group in forced vital capacity. 


\section{Respiratory muscle strength}

\section{Maximal inspiratory pressure}

Three studies with a total of 244 participants evaluated MIP as an outcome $[8,16,18]$. A total of 119 participants received IMT and 125 participants formed the control group. The heterogeneity $\left(\mathrm{I}^{2}\right)$ was $69 \%\left(\mathrm{P}_{\text {Heterogeneity }}=0.53\right)$. These studies reported that there was an improvement in MIP in the intervention group compared to that in the control group, with a mean difference of $4.70 \mathrm{cmH}_{2} \mathrm{O}(95 \%$ CI, -4.00 to 13.4) (Fig. 5).

\section{Maximal expiratory pressure}

One study, which included 10 participants, documented the effects of IMT on maximal expiratory pressure [18]. There was a difference of $-2.1 \mathrm{cmH}_{2} \mathrm{O}$ that favored the control group.

\section{Length of hospital stay}

Four studies included in the review measured the length of hospital stay as an outcome $[8,16-18]$. The intervention and control groups included 132 and 137 participants, respectively. There was a mean difference of - 1.82 days ( $95 \%$ CI, -2.45 to -1.18). The length of hospital stay of participants given IMT, either preoperatively or postoperatively, was significantly shorter than that of participants in the control group (Fig. 6).

\section{DISCUSSION}

To our knowledge, this is the first systematic review and

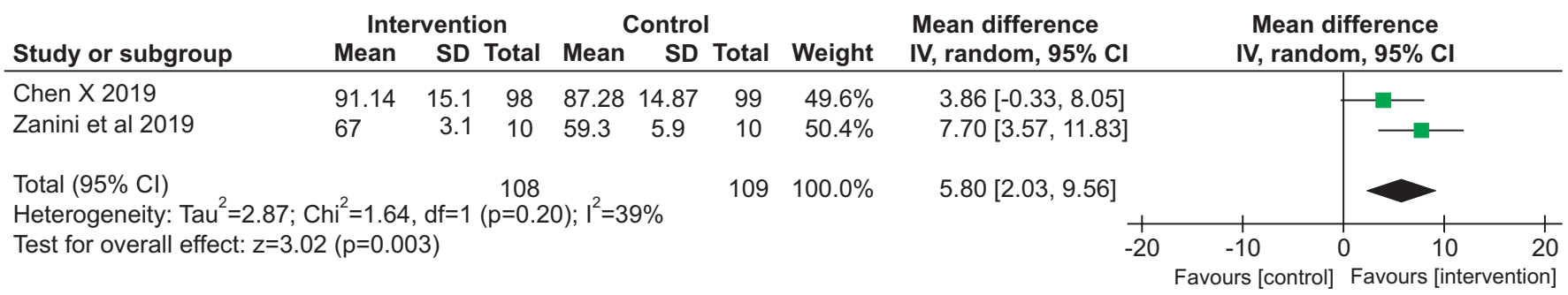

Fig. 4. Forest plot comparing the intervention group versus control group in forced expiratory volume in 1 second.

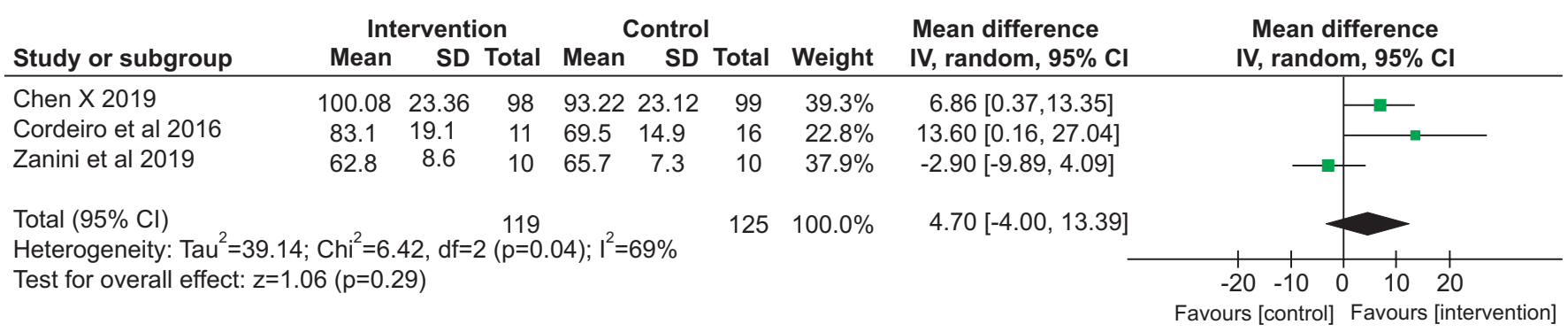

Fig. 5. Forest plot comparing the intervention group versus control group in maximal inspiratory pressure.

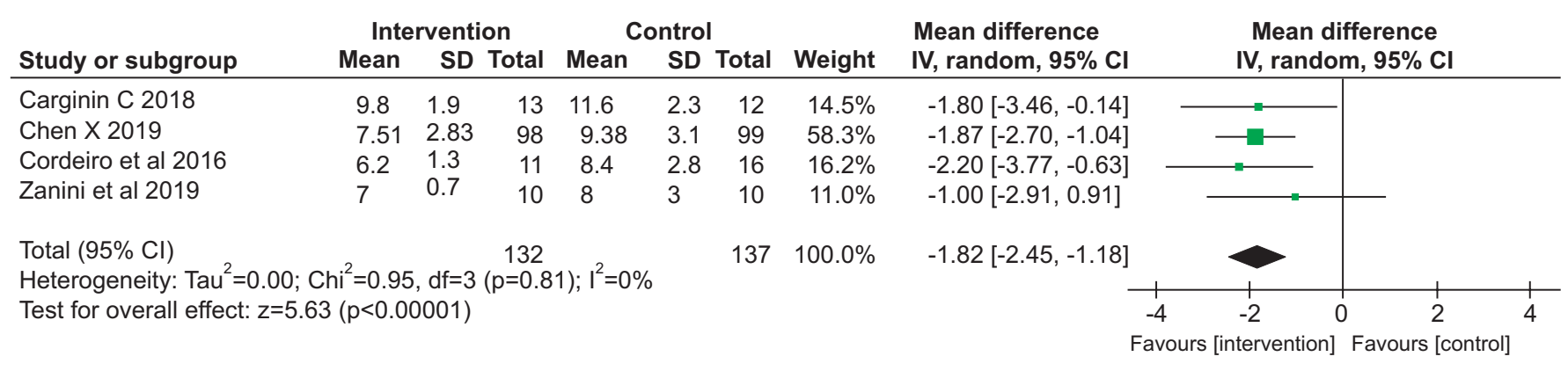

Fig. 6. Forest plot comparing the intervention group versus control group in length of hospital stay. 
meta-analysis to examine the effect of IMT in patients undergoing cardiac surgeries. The results of the metaanalysis suggest that there is a remarkable improvement in RMS, functional capacity, and lung function. However, the included studies showed heterogeneity in variables such as training period (preoperative IMT or postoperative IMT) and duration of sessions. Only one study used IMT in the preoperative phase. Dos Santos et al. [3] reported on IMT in the second phase of cardiac rehabilitation, whereas in three studies, it was provided during the first phase of cardiac rehabilitation. The first phase of cardiac rehabilitation refers to rehabilitation during hospitalization. The second phase includes rehabilitation up to 4 months after discharge from the hospital and consists of monitored outpatient physical activity as recommended by the physician. Subsequently, the third phase of cardiac rehabilitation involves a permanent, unmonitored exercise program [19]. We conducted a meta-analysis of all included studies, which indicated an improvement in RMS, functional capacity as measured by the 6-minute walk distance, and lung function.

RMS was evaluated as the primary outcome in this systematic review and meta-analysis. RMS is reduced in all individuals undergoing cardiac surgery. Previous studies have shown that neural conditioning caused by repeated exposure to the same task improves RMS by improving neuromuscular recruitment patterns [8]. According to Zanini et al. [18], RMS improves by the 30th postoperative day and that there is no significant improvement in the IMT group compared to other groups. Moreover, reversal of RMS has been reported due to the additional effect of expiratory positive airway pressure and not IMT alone. However, Cordeiro et al. [16] noted better RMS at the time of discharge in a population who underwent IMT post-surgery. This difference could be due to the higher resistance used by Cordeiro et al. [16] or the difference in the population. Meta-analysis reported the highest heterogeneity, $\mathrm{I}^{2}=69 \%$.

De Menezes et al. [20] reported that reduced RMS was associated with decreased functional capacity and an increased period of recovery of lung function, as it is directly related to peripheral muscle strength. Therefore, improvement in RMS can improve FVC and $\mathrm{FEV}_{1}$ and thereby the 6-minute walking distance. We included two studies to analyze the effects of IMT on FVC and $\mathrm{FEV}_{1}$. A meta-analysis of both studies showed an improvement in FVC, irrespective of whether IMT was administered preoperatively or postoperatively. Nevertheless, the study by Zanini et al. [18], which assessed postoperative intervention, reported better improvement than the study by Chen et al. [8]. The study by Shenkman et al. [21] reported that individuals with CABG had a $70 \%$ reduction in FVC immediately postoperatively and a reduction of $35 \%$ at 3 weeks after surgery.

PPCs usually prolong the postoperative length of hospital stay. Previous studies by Hulzebos et al. [22] reported that preoperative IMT reduced the incidence of PPCs. In addition, a systematic review and meta-analysis conducted by Gomes et al. [23] showed similar findings. In our review, only the study by Chen et al. [8] reported the reduction of PPCs after preoperative IMT, but other studies have not focused on this aspect.

Four studies with no heterogeneity $\left(\mathrm{I}^{2}=0 \%\right)$ were used for meta-analysis of the length of hospital stay. The metaanalysis indicated that there was a significant improvement in the intervention group compared to the control group. A reduction in the length of stay can be associated with a reduction in PPCs in the intervention group. Improved inspiratory muscle strength improves the efficacy of cough, and thereby, clearance of secretions and reduction in PPCs.

This systematic review and meta-analysis has a few limitations. Only five articles, with intervention administered either preoperatively or postoperatively, were eligible for the systematic review. We were unable to compare studies with preoperative IMT with those with postoperative IMT, as very few studies with independent preoperative IMT were available. The sample size was small, and one study included data from only males in the interpretation of results [16]. In addition, we included studies in which intervention was provided at different time intervals. Furthermore, differences in exercise intensities and duration of sessions may have also contributed to the heterogeneity, which was unavoidable as there are very few studies on this subject. Hence, further studies are required to determine whether IMT helps improve RMS, functional capacity, and pulmonary function.

In conclusion, the authors recommend the use of IMT, either before or after cardiac surgery, as it successfully improves pulmonary function, inspiratory muscle strength, functional capacity, and duration of hospitalization. 


\section{CONFLICT OF INTEREST}

No potential conflict of interest relevant to this article was reported.

\section{AUTHOR CONTRIBUTION}

Conceptualization: Kumar S, Dsouza F, Samuel S. Methodology: Dsouza F. Formal analysis: Ravishankar N, Dsouza, Kumar S, Samuel S. Project administration: Kumar S. Visualization: Samuel S, Kumar S, Raghavan H. Writing-original draft: Dsouza F, Kumar S. Writing-review and editing: Kumar S, Dsouza F, Samuel S. Approval of final manuscript: all authors.

\section{REFERENCES}

1. Gupta R, Mohan I, Narula J. Trends in coronary heart disease epidemiology in India. Ann Glob Health 2016;82:307-15.

2. GBD 2016 Causes of Death Collaborators. Global, regional, and national age-sex specific mortality for 264 causes of death, 1980-2016: a systematic analysis for the Global Burden of Disease Study 2016. Lancet 2017;390:1151-210.

3. Dos Santos TD, Pereira SN, Portela LO, Cardoso DM, Lago PD, Dos Santos Guarda N, et al. Moderate-tohigh intensity inspiratory muscle training improves the effects of combined training on exercise capacity in patients after coronary artery bypass graft surgery: a randomized clinical trial. Int J Cardiol 2019;279:406.

4. Sibilitz KL, Berg SK, Hansen TB, Risom SS, Rasmussen TB, Hassager C, et al. Effect of comprehensive cardiac rehabilitation after heart valve surgery (CopenHeart VR): study protocol for a randomised clinical trial. Trials 2013;14:1-14.

5. Haeffener MP, Ferreira GM, Barreto SS, Arena R, Dall'Ago P. Incentive spirometry with expiratory positive airway pressure reduces pulmonary complications, improves pulmonary function and 6-minute walk distance in patients undergoing coronary artery bypass graft surgery. Am Heart J 2008;156:900.e1-900. e8.

6. Wynne R, Botti M. Postoperative pulmonary dysfunction in adults after cardiac surgery with cardiopulmo- nary bypass: clinical significance and implications for practice. Am J Crit Care 2004;13:384-93.

7. Shakouri SK, Salekzamani Y, Taghizadieh A, SabbaghJadid H, Soleymani J, Sahebi L, et al. Effect of respiratory rehabilitation before open cardiac surgery on respiratory function: a randomized clinical trial. J Cardiovasc Thorac Res 2015;7:13-7.

8. Chen X, Hou L, Zhang Y, Liu X, Shao B, Yuan B, et al. The effects of five days of intensive preoperative inspiratory muscle training on postoperative complications and outcome in patients having cardiac surgery: a randomized controlled trial. Clin Rehabil 2019;33:91322.

9. Baumgarten MC, Garcia GK, Frantzeski MH, Giacomazzi CM, Lagni VB, Dias AS, et al. Pain and pulmonary function in patients submitted to heart surgery via sternotomy. Rev Bras Cir Cardiovasc 2009;24:497-505.

10. Praveen R, Swaminathan N, Praveen JS. Inspiratory muscle training is effective in improving respiratory muscle functions in patients who have undergone coronary artery bypass graft. Fizjoterapia Polska 2009;9:285-92.

11. Siafakas NM, Mitrouska I, Bouros D, Georgopoulos D. Surgery and the respiratory muscles. Thorax 1999;54:458-65.

12. Westerdahl E, Jonsson M, Emtner M. Pulmonary function and health-related quality of life 1-year follow up after cardiac surgery. J Cardiothorac Surg 2016;11:99.

13. Westerdahl E, Lindmark B, Bryngelsson I, Tenling A. Pulmonary function 4 months after coronary artery bypass graft surgery. Respir Med 2003;97:317-22.

14. Mans CM, Reeve JC, Elkins MR. Postoperative outcomes following preoperative inspiratory muscle training in patients undergoing cardiothoracic or upper abdominal surgery: a systematic review and meta analysis. Clin Rehabil 2015;29:426-38.

15. Savci S, Degirmenci B, Saglam M, Arikan H, Inal-Ince D, Turan HN, et al. Short-term effects of inspiratory muscle training in coronary artery bypass graft surgery: a randomized controlled trial. Scand Cardiovasc J 2011;45:286-93.

16. Cordeiro AL, de Melo TA, Neves D, Luna J, Esquivel MS, Guimaraes AR, et al. Inspiratory muscle training and functional capacity in patients undergoing cardiac surgery. Braz J Cardiovasc Surg 2016;31:140-4. 
17. Cargnin C, Karsten M, Guaragna JC, Dal Lago P. Inspiratory muscle training after heart valve replacement surgery improves inspiratory muscle strength, lung function, and functional capacity: a randomized controlled trial. J Cardiopulm Rehabil Prev 2019;39:E1E7.

18. Zanini M, Nery RM, de Lima JB, Buhler RP, da Silveira AD, Stein R. Effects of different rehabilitation protocols in inpatient cardiac rehabilitation after coronary artery bypass graft surgery: a randomized clinical trial. J Cardiopulm Rehabil Prev 2019;39:E19-E25.

19. McMahon SR, Ades PA, Thompson PD. The role of cardiac rehabilitation in patients with heart disease. Trends Cardiovasc Med 2017;27:420-5.

20. De Menezes TC, Bassi D, Cavalcanti RC, Barros JE, Granja KS, Calles AC, et al. Comparisons and correla- tions of pain intensity and respiratory and peripheral muscle strength in the pre- and postoperative periods of cardiac surgery. Rev Bras Ter Intensiva 2018;30:47986.

21. Shenkman Z, Shir Y, Weiss YG, Bleiberg B, Gross D. The effects of cardiac surgery on early and late pulmonary functions. Acta Anaesthesiol Scand 1997;41:11939.

22. Hulzebos EH, Smit Y, Helders PP, van Meeteren NL. Preoperative physical therapy for elective cardiac surgery patients. Cochrane Database Syst Rev 2012;11:CD010118.

23. Gomes Neto M, Martinez BP, Reis HF, Carvalho VO. Pre- and postoperative inspiratory muscle training in patients undergoing cardiac surgery: systematic review and meta-analysis. Clin Rehabil 2017;31:454-64. 